\title{
OPEN ACCESS - GANZ, TEILWEISE ODER GAR NICHT: WIE OFFEN IST DER ZUGANG ZU DEN DEUTSCHSPRACHIGEN ZEIT- SCHRIFTEN FÜR BIBLIOTHEK UND INFORMATION?
}

\author{
von Otto Oberhauser
}

\author{
Inhalt \\ 1. Einleitung \\ 2. Reine Online-Zeitschriften mit vollständigem offenen Zugang \\ 3. Hybride Zeitschriften mit vollständigem offenen Zugang \\ 4. Zeitschriften mit zeitverzögertem offenen Zugang \\ 5. Zeitschriften mit teilweisem offenen Zugang \\ 6. Schlussbemerkungen
}

Zusammenfassung: Der Beitrag untersucht, inwieweit die im deutschsprachigen Raum erscheinenden Zeitschriften für das Fachgebiet Bibliotheks- und Informationswesen offen zugänglich sind. Die betreffenden Journale werden identifiziert und besprochen. Der Autor differenziert dabei das Angebot nach den Kategorien reine Online-Zeitschriften mit vollständigem offenen Zugang, hybride Zeitschriften mit vollständigem offenen Zugang, Zeitschriften mit zeitverzögertem offenen Zugang sowie Zeitschriften mit teilweisem offenen Zugang. Die Anzahl der bibliothekarischen bzw. informationswissenschaftlichen Fachjournale ohne zumindest teilweise oder zeitverzögerte freie Zugänglichkeit ist im deutschsprachigen Raum erfreulicherweise sehr gering.

Schlüsselwörter: Deutschland; Österreich; Schweiz; deutschsprachiger Raum; Fachzeitschriften; Bibliothekswesen; Informationswesen; freie Zugänglichkeit; Open Access; Übersicht; Kategorisierung

\section{OPEN ACCESS - TOTALLY, PARTIALLY OR NOT AT ALL: HOW OPEN IS THE ACCESS TO THE GERMAN-LANGUAGE JOURNALS IN THE AREA OF LIBRARY AND INFORMATION SCIENCE?}

Abstract: The article examines the extent to which the journals in the area of library and information science in German-speaking countries are openly accessible. The journals in question are identified and discussed. The author differentiates the relevant publications by categories such as online-only journals with full open access, hybrid journals with full open access, journals with time-delayed open access, as well as magazines with partial open access. The number of library and information science journals without at least partial or delayed free access is fortunately very low in the German-speaking area. 
Keywords: Germany; Austria; Switzerland; German-speaking area; professional journals; library and information science; overview; categorization; Open Access

\section{Einleitung}

Gemäss der weit verbreiteten Definition der Budapest Open Access Initiative (BOAI) meint Open Access (OA), „dass [= Peer-Review-Fachliteratur] kostenfrei und öffentlich im Internet zugänglich sein sollte, sodass Interessenten die Volltexte lesen, herunterladen, kopieren, verteilen, drucken, in ihnen suchen, auf sie verweisen und sie auch sonst auf jede denkbare legale Weise benutzen können, ohne finanzielle, gesetzliche oder technische Barrieren (... "1 Diese allgemeine Definition schliesst die verschiedensten Publikationsformen ein. Wenn es um eine Definition von "Open-AccessZeitschriften" geht, bietet das DOAJ (Directory of Open Access Journals) einen Einstieg: „We define open access journals as journals that use a funding model that does not charge readers or their institutions for access." Weitere Kriterien, so erfahren wir dort auch, sind a) das Vorhandensein einer Qualitätskontrolle (peer-review oder editorial review), b) die Publikation primär von Forschungsergebnissen oder -überblicken für eine wissenschaftliche Leserschaft, und c) der periodische Charakter, was laut DOAJ bedeutet, dass die Zeitschrift öfter als einmal pro Jahr erscheinen muss. Ausserdem muss eine Zeitschrift mindestens fünf ,research and review articles" pro Jahr veröffentlichen, um im DOAJ gelistet zu bleiben. ${ }^{3}$ Dass es sich um elektronische Zeitschriften handeln muss, wird gar nicht explizit formuliert, wohl aber vorausgesetzt.

Ob man mit allen Kriterien des DOAJ einverstanden sein soll, ist durchaus in Frage zu stellen, wenn es - wie hier - um den freien Zugang zu den Zeitschriften des Fachgebiets Bibliotheks- und Informationswesen geht. Streng genommen gäbe es da nämlich kaum relevante Titel, da wohl nur eine Minderheit der in unseren Zeitschriften publizierten Beiträge Forschungsergebnisse im engeren Sinn enthält - vielmehr handelt es sich mehrheitlich um Fachartikel verschiedenster Art. Auch die Leserschaft ist weniger eine wissenschaftliche als eine professionelle. Zudem wird das Reviewing wahrscheinlich nicht in jedem Fall strengen Kriterien genügen. Schliesslich wäre es auch nicht sinnvoll, eine Publikation von der Betrachtung auszuschliessen, die durchaus zeitschriftenartig anmutet, aber nur einmal im Jahr erscheint bzw. keine fünf „research and review articles“ aufweist.

Ein weiteres wichtiges Kriterium wird auf der Informationsplattform open-access.net betont: „Open-Access-Zeitschriften sind Zeitschriften, deren 
Artikel unmittelbar mit Erscheinen der Zeitschrift kostenlos und frei von weiteren Einschränkungen weltweit zugänglich sind. Beiträge in Zeitschriften, die dieser Definition entsprechen, erscheinen in der Regel unter einer freien Lizenz, die durch die Autor/innen vergeben wird; auf einen CopyrightTransfer an die Zeitschrift verzichten diese Zeitschriften. Gleichzeitig gibt es viele Zeitschriften, die ihre Inhalte zur kostenlosen Nutzung bereitstellen, aber keine freie Lizenz vergeben. " ${ }^{4}$ Und an anderer Stelle wird dort erläutert: „Anhand von Open-Content-Lizenzen kann der Autor/die Autorin festlegen, zu welchen Bedingungen ein Open Access bereitgestelltes Dokument von Dritten genutzt werden kann. Die Einräumung bestimmter Nutzungsrechte anhand solcher Lizenzen vereinfacht die Rechtsdurchsetzung bei Missbrauch und gibt den Nutzerinnen und Nutzern explizite Hinweise darauf, wie das Dokument weiter verwendet werden darf. Gleichzeitig behält der Autor/die Autorin die Möglichkeit, weitere, über die durch die Lizenz hinausgehende Nutzungen in gesonderten Verträgen zu erlauben. “5 Es wird daher in diesem Beitrag, wiewohl sich dieser primär mit der freien Zugänglichkeit von Zeitschriften für das Fachpublikum beschäftigt, auch stets ein Blick auf die Lizenzfrage zu werfen sein.

Dass die Strenge des obigen Statements dem Spektrum der OA-Zeitschriften nicht ganz gerecht wird, zeigt auch ein Blick in den diesbezüglichen Eintrag der englischsprachigen Wikipedia, ${ }^{6}$ wo Begriffe wie „hybrid open access journals" und „delayed open access journals" genannt werden, die zu einer Typologie führen, die der Realität viel näher kommt: „In successively looser senses, open access journals may be considered as:

- Journals entirely open access

- Journals with research articles open access (hybrid open access journals)

- Journals with some research articles open access (hybrid open access journals)

- Journals with some articles open access and the other delayed access

- Journals with delayed open access (delayed open access journals)

- Journals permitting self-archiving of articles. "7

Neben Zeitschriften mit vollständigem offenen Zugang gibt es also auch solche, bei denen sich dieser auf bestimmte Artikel beschränkt und/oder erst nach Ablauf einer Sperrfrist (Embargozeitraum, Moving Wall) möglich ist. Am unteren Ende der Skala werden schliesslich auch Zeitschriften angeführt, die bloss eine Selbstarchivierung durch die Autor/innen in (frei zugänglichen) Repositorien gestatten. 
Im folgenden wird die mehr oder weniger offene Zugänglichkeit der deutschsprachigen Fachzeitschriften des Bibliotheks- und Informationswesens untersucht. Der Beitrag soll vor allem dazu dienen, der interessierten Leserschaft zu zeigen, was wo und wann für sie frei verfügbar ist, und so vielleicht auch zu einem gesteigerten Interesse an der Nutzung der betreffenden Journale beitragen. Um die Darstellung nicht ausufern zu lassen, bleiben solche Zeitschriften unberücksichtigt, a) die nur gelegentlich deutschsprachige Beiträge bringen, b) die sich ausschliesslich auf die Welt der öffentlichen Bibliotheken (Büchereien) beziehen, $c$ ) die sich nur als (semi-)internes Organ einer einzelnen Bibliothek begreifen, d) die reine Newsletters sind. Als Quelle für die Auswahl diente vor allem die Gruppe „Informations-, Buch- und Bibliothekswesen, Schrift- und Handschriftenkunde" der Elektronischen Zeitschriftenbibliothek EZB. ${ }^{8}$

\section{Reine Online-Zeitschriften mit vollständigem offenen Zugang}

Die Gruppe der Zeitschriften mit vollständigem offenen Zugang lässt sich weiter in zwei Kategorien unterteilen: a) reine Online-Zeitschriften und b) solche Journale, die parallel zu einer frei zugänglichen Online-Version eine gedruckte Ausgabe zur Verfügung stellen (s.a. Tabelle 1). Journale aus beiden Kategorien wurden im Vorjahr in dieser Zeitschrift vorgestellt, ${ }^{9}$ wobei aber nur eine Auswahl getroffen wurde. Gleichwohl braucht das dort Gesagte hier nicht im Detail wiederholt werden. ${ }^{10}$ Dies betrifft in der ersten Kategorie die beiden etablierten Online-Zeitschriften GMS Medizin - Bibliothek - Information und LIBREAS. Library ideas sowie die beiden neueren Journale Perspektive Bibliothek und 027.7: Zeitschrift für Bibliothekskultur. Die Beiträge in diesen Publikationen stehen unter den Creative Commons Lizenzbedingungen „Namensnennung 4.0 International (CC BY 4.0)“ bzw. „Namensnennung 3.0 Unported (CC BY 3.0)“; alle vier sind auch im DOAJ gelistet. Die beiden letztgenannten Zeitschriften werden unter Verwendung des Zeitschriftenmanagement- und -publikationssystems Open Journal Systems (OJS) produziert.

Die beiden im vorjährigen Beitrag erst angekündigten OA-Zeitschriften haben inzwischen ihr Erscheinen aufgenommen. Gerade noch vor Weihnachten 2014 wurde die erste Ausgabe der neuen Zeitschrift des Vereins Deutscher Bibliothekare (VDB), o-bib: Das offene Bibliotheksjournal, freigeschaltet. ${ }^{11}$ Die vom Herausgeber als «Null-Nummer» bezeichnete, de facto aber als „Bd. 1, Nr. 1 (2014)“ gezählte Ausgabe enthält überwiegend ausgewählte Beiträge des 103. Deutschen Bibliothekartages in Bremen. Neben 
einem Editorial und der die Bibliothekartagsvorträge umfassenden Rubrik „Kongressbeiträge“ (begutachtet) enthält das erste Heft auch noch die Rubriken „Tagungsberichte“ und „Aus dem VDB“, mit der die bisher auch online erschienenen VDB-Mitteilungen abgelöst wurden. ${ }^{12}$ Ab 2015 soll o-bib mit vier Ausgaben pro Jahr erscheinen, deren erste bereits vorliegt. Sie weist als neue Rubriken „Aufsätze / Fachbeiträge“ (begutachtet) und „Rezensionen" auf; zukünftig sind noch Rubriken für Diskussionen, Personalia und Mitteilungen bzw. kleinere Beiträge geplant. Die Beiträge der begutachteten Rubriken unterliegen grundsätzlich einem Peer Review durch zwei Gutachter. In inhaltlicher Hinsicht ist $o$-bib auf das wissenschaftliche Bibliothekswesen ausgerichtet.

Das Konzept der zweiten im Vorjahr angekündigten Zeitschrift, der von einem Schweizer Verein getragenen und spartenübergreifend ausgerichteten Informationspraxis, sieht vor, dass die redaktionell freigegebenen Beiträge unmittelbar veröffentlicht, halbjährlich aber zu einer virtuellen Ausgabe zusammengefasst werden. Da die Zeitschrift auch ein Open Peer Review-Verfahren erprobt, konnte man die letztlich in der ersten Ausgabe erschienenen Artikel schon einige Zeit zuvor als Preprints sehen und gegebenenfalls auch kommentieren. Die bisherigen Erfahrungen mit diesem Reviewsystem scheinen allerdings, soweit man dies aus Blogeinträgen ersehen kann, eher gemischt zu sein. Wünscht die Autorin/der Autor kein Open Peer Review, so ist jedenfalls ein traditionelles Review durch eine Gutachterin/einen Gutachter vorgesehen, wofür auch Richtlinien publiziert wurden. Die erste Ausgabe - sie wird als Bd. 1, Nr. 1 (2015) geführt und als „public beta“ bezeichnet - enthält neben einem Editorial die Rubriken „Fachbeiträge“ und „Kurzberichte“ (beide begutachtet). Die Zeitschrift verfügt bereits über ein Editorial Board von beachtlichem Umfang, sodass für traditionelle Reviews ein grosses Potenzial fachlich spezialisierter Gutachter zur Verfügung steht. Informationspraxis verwendet, wie auch o-bib, OJS als Publikationssystem. Die Beiträge beider Zeitschriften erscheinen unter der Lizenz CC BY 4.0. Beide Journale sind (noch) nicht im DOAJ gelistet.

Unter den reinen Online-Journalen sind noch zwei Zeitschriften zu erwähnen. Braln: Potsdamer Beiträge und Reportagen aus der Informationswissenschaft ist die frei zugängliche elektronische Zeitschrift des Fachbereichs Informationswissenschaften der FH Potsdam. Sie entstand 2008 im Rahmen eines studentischen Projekts am Fachbereich und wird seitdem unter der Obhut des den betreffenden Projektkurs leitenden Professors mit semesterweise wechselnden Redaktionsteams weitergeführt. Braln veröffentlicht Forschungsberichte des Lehrkörpers, Essays und herausragende Beiträge 
der Studierenden wie zum Beispiel Haus-, Seminar- und Abschlussarbeiten sowie Exkursions- und Praktikumsberichte. Ein Redaktionsbeirat, bestehend aus Studierenden, sorgt für die Qualitätskontrolle, was wohl als editorial review gelten kann. ${ }^{13}$ Prüfungsarbeiten werden darüber hinaus von den Lehrenden begutachtet und der Redaktion empfohlen. Die Zeitschrift erscheint zweimal jährlich im HTML-Format; ursprünglich wurde jeder Beitrag zusätzlich auch als PDF-Datei zur Verfügung gestellt, was jedoch offenbar bald wieder aufgegeben wurde. Braln ist im DOAJ gelistet, scheint dort aber nur unter dem Untertitel Potsdamer Beiträge und Reportagen aus der Informationswissenschaft auf. Die Beiträge erscheinen frei zugänglich, allerdings ohne CC-Lizenz.

Beim zweiten Titel handelt es sich um das anlässlich einer Fachkonferenz 2012 an der UB Regensburg begründete Journal Bibliometrie - Praxis und Forschung, das sich selbst als ,erste deutsche Online-Fachzeitschrift zu bibliometrischen Verfahren" bezeichnet. Nach der Definition des DOAJ würde es sich aber gar nicht um eine Zeitschrift handeln, da bisher pro Jahr stets nur eine Ausgabe erschienen ist und auch die Erfüllung des 5-Artikel-Kriteriums in Frage steht. Spitzfindigkeiten dieser Art sind aber wenig hilfreich, denn - so wurde bereits in der Einleitung angemerkt - das auf Basis des OJS verfertigte Journal versteht sich nicht nur selbst als Zeitschrift, sondern sieht auch wie eine solche aus und wird vielleicht auch einmal mehr als nur eine Ausgabe pro Jahr aufweisen. Die Qualitätssicherung der Beiträge erfolgt je nach Rubrik durch das Editorial Board bzw. durch Peer Reviewer. Bisher sind neben „Editorial“ die Rubriken „Konferenzbeiträge“, „Artikel“ und „Tagungsberichte“ verwendet worden. Bei der Mehrheit der seit 2012 erschienenen Beiträge handelt es sich um Konferenzvorträge. Im Impressum wird vermerkt, dass alle Beiträge, die übrigens sämtlich ein Creative Commons-Logo tragen, frei nachnutzbar sind. Nicht angegeben sind allerdings die Version der CC BY Lizenz und die Verlinkung zum Lizenztext.

\section{Hybride Zeitschriften mit vollständigem offenen Zugang}

Die zweite Kategorie umfasst jene Zeitschriften, die parallel zu einer - meist kostenpflichtigen - gedruckten Ausgabe auch eine frei zugängliche OnlineVersion anbieten (s.a. Tabelle 1) und daher als „hybrid“14 bezeichnet werden. Aus naheliegenden Gründen sind hier zuvorderst die Mitteilungen der Vereinigung Österreichischer Bibliothekarinnen \& Bibliothekare anzuführen, die schon in dem 2014 publizierten Artikel besprochen wurden und deshalb 


\begin{tabular}{|c|c|c|c|c|c|c|c|c|c|c|c|c|c|c|c|}
\hline 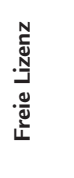 & 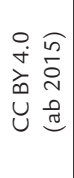 & $\begin{array}{l}\stackrel{0}{m} \\
\grave{\infty} \\
\cup\end{array}$ & $\begin{array}{l}\circ \\
\dot{+} \\
\text { ذे } \\
\cup \\
u\end{array}$ & $\begin{array}{l}\stackrel{0}{m} \\
\grave{\infty} \\
\dot{u}\end{array}$ & $\begin{array}{l}\circ \\
\dot{+} \\
\grave{\infty} \\
\cup \\
\cup\end{array}$ & 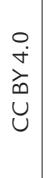 & 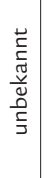 & $\cup$ & 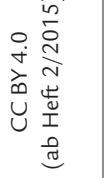 & : & : & 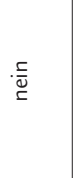 & · & : & : \\
\hline 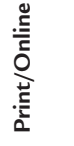 & 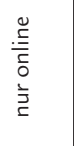 & 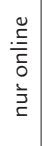 & 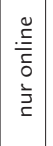 & 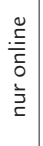 & 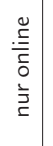 & 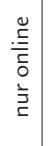 & 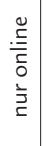 & 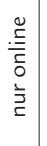 & 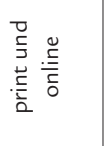 & 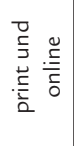 & 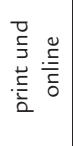 & 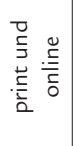 & 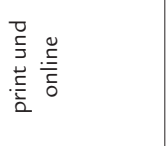 & 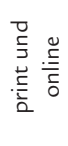 & 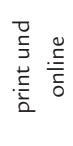 \\
\hline 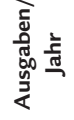 & $m$ & $\stackrel{m}{\sim}$ & $\sim$ & $\stackrel{m}{1}$ & $\nabla$ & $N$ & $N$ & - & $\begin{array}{l}+ \\
\text { m }\end{array}$ & $\sim$ & $\nabla$ & $m$ & $m$ & $\nabla$ & $m$ \\
\hline 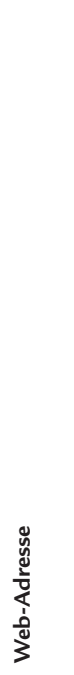 & 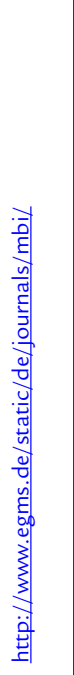 & 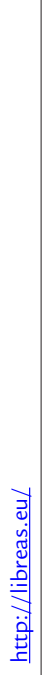 & 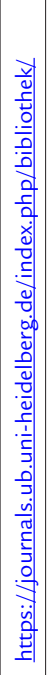 & 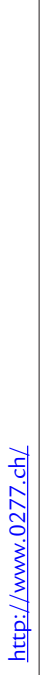 & 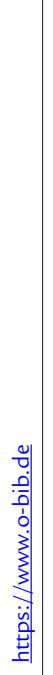 & 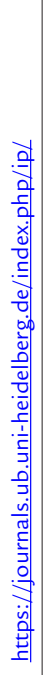 & 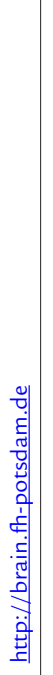 & 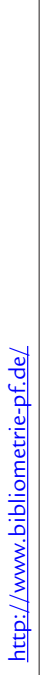 & 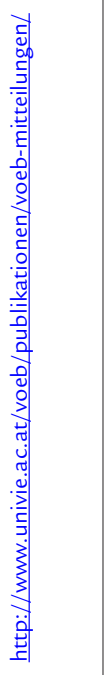 & 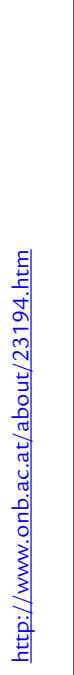 & 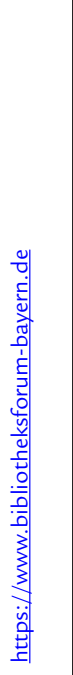 & 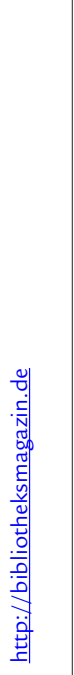 & 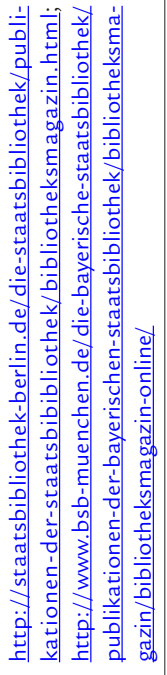 & 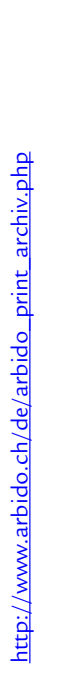 & 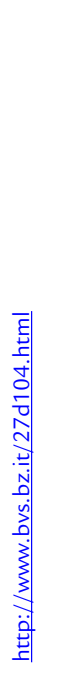 \\
\hline 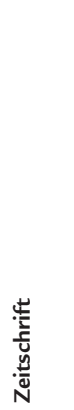 & 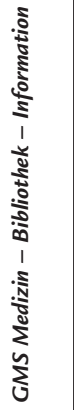 & 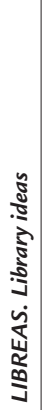 & 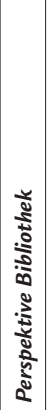 & $\stackrel{\hat{N}}{\hat{\sigma}}$ & ठี & 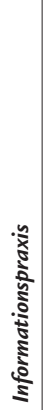 & है & 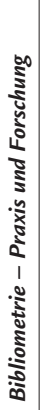 & 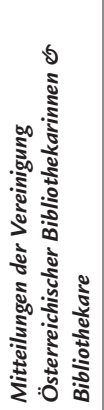 & $\stackrel{\text { Oे }}{\frac{5}{00}}$ & 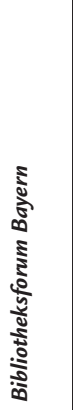 & $\frac{n}{\infty}$ & 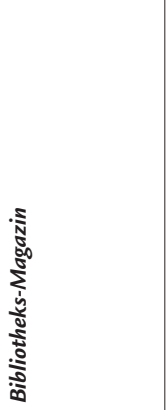 & $\frac{10}{8}$ & 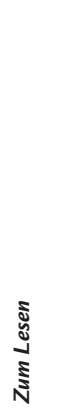 \\
\hline
\end{tabular}

Tab. 1: Zeitschriften mit vollständigem offenen Zugang (online und hybrid) 
hier nur gestreift werden sollen. Erwähnt seien neben der freien Zugänglichkeit der Online-Ausgabe die seit längerem gut eingespielte Praxis des Peer Review-Verfahrens, die Verwendung der Creative Commons Lizenz „Namensnennung 3.0 Österreich (CC BY 3.0 AT)“ (bis Heft 1/2015) bzw. „Namensnennung 4.0 International (CC BY 4.0)" (ab Heft 2/2015) für alle Beiträge, sowie die redaktionelle Einspeicherung sämtlicher Artikel in das Repositorium E-LIS. Die Zeitschrift ist (noch) nicht im DOAJ gelistet und verwendet derzeit noch nicht, aber möglicherweise bald, OJS als Veröffentlichungsplattform.

Seit kurzem ist auch die traditionsreiche Zeitschrift der Österreichischen Nationalbibliothek, Biblos: Beiträge zu Buch, Bibliothek und Schrift, parallel zu der im Wiener Phoibos Verlag erscheinenden Druckausgabe in einer frei zugänglichen Online-Version verfügbar. Bei dieser handelt es sich um das jeweils ganze Zeitschriftenheft in Form einer PDF-Datei. Die beiden bisher online frei zugänglichen Hefte (Jahrgang 2014) unterlagen keiner Sperrfrist. Die in der Regel zu Schwerpunktthemen verfassten Beiträge werden durch ein neunköpfiges Redaktionsteam begutachtet und unterliegen keiner freien Lizenz.

Das Bibliotheksforum Bayern (BFB) ist die Fachzeitschrift des Bibliotheksverbunds Bayern. Sie entstand 2007 durch die Zusammenführung zweier voneinander sehr verschiedener Vorgängerzeitschriften - Öffentliche Bibliotheken in Bayern (ÖBiB) für das öffentliche und Bibliotheksforum Bayern (BFB) für das wissenschaftliche Bibliothekswesen - zu einer gemeinsamen Fachzeitschrift für die beiden Sparten, die Fachbeiträge und Nachrichten bringt. Neben der viermal pro Jahr erscheinenden Druckausgabe wird BFB gleichzeitig auf einem Server der Bayerischen Staatsbibliothek kostenlos online zur Verfügung gestellt. Die sichtlich nicht mit OJS produzierte Online-Ausgabe lässt einerseits ein komfortables Blättern in der Zeitschrift und andererseits die Navigation über das Inhaltsverzeichnis zu, mit der die Beiträge als PDF-Files aufgerufen bzw. heruntergeladen werden können. Ein Redaktionsteam (vier Personen) und ein Redaktionsbeirat (sieben Personen) gestalten das inhaltliche und konzeptionelle Profil der Zeitschrift. Das Reviewing der unter keiner freien Lizenz stehenden Beiträge erfolgt offenbar redaktionell.

BIS: Das Magazin der Bibliotheken in Sachsen ist ein seit 2008 bestehendes Gemeinschaftswerk der öffentlichen und wissenschaftlichen Bibliotheken im Freistaat Sachsen. Das Magazin berichtet über Sammlungen und Häuser, innovative Angebote und beispielhafte Dienste, und wendet sich an einen breiten Leserkreis. Es ist an der Sächsischen Landesbibliothek - Staatsund Universitätsbibliothek Dresden (SLUB) angesiedelt, die es gemeinsam 
mit anderen Institutionen herausgibt. BIS erscheint dreimal jährlich in einer Druckausgabe; zugleich wird das Journal kostenlos auf der DINI-zertifizierten OA-Plattform der SLUB, Qucosa, ${ }^{15}$ zur Verfügung gestellt, wobei es einerseits möglich ist, das komplette Heft als PDF-Datei abzuspeichern, andererseits aber auch, die einzelnen Artikel über eine Inhaltsübersicht anzusteuern. Dabei wird auf eine Webseite verlinkt, die Titel, Abstract und weitere Metadaten aufweist und von der aus dann auch der betreffende Volltext im PDF-Format geöffnet werden kann.Neben dem Editorial und den Artikeln bringt BIS auch Kurzmeldungen. Das Redaktionsteam umfasst derzeit fünf Personen (alle SLUB); daneben besteht ein sechsköpfiger Redaktionsbeirat. Über Peer Review bzw. freie Lizenz ist der Website von $B I S$ nichts zu entnehmen.

Aus Deutschland kommt schliesslich auch das Journal Bibliotheks-Magazin: Mitteilungen aus den Staatsbibliotheken in Berlin und München, das dreimal jährlich über die Sammlungen, Aktivitäten und Dienstleistungen der beiden grossen Universalbibliotheken berichtet. Mit der gedruckten Ausgabe erscheint gleichzeitig auch eine kostenlose Online-Version, bei der es sich allerdings nur um eine PDF-Datei handelt, die das ganze Heft beinhaltet. Diese Online-Ausgabe ist von den Webseiten beider Bibliotheken aus erreichbar (vgl. Tab. 1). Die Zeitschrift wird von Redaktionen in Berlin und München in grafisch ansprechender, bildreicher Form gestaltet. Da sie sowohl eine fortlaufende Zählung als auch eine solche mit Jahrgang und Heftnummer aufweist, ${ }^{16}$ ist die Zitierung der Beiträge nicht ganz unproblematisch. Über die Begutachtung der Artikel bzw. freie Lizenzen geht aus dem Webauftritt nichts Näheres hervor.

Die Zeitschrift arbido ist das gemeinsame Publikationsorgan der beiden Berufsverbände Bibliothek Information Schweiz (BIS) und Verein Schweizerischer Archivarinnen und Archivare (VSA-AAS). Die Zeitschrift wendet sich an alle, die in Archiven, Bibliotheken, Dokumentationsstellen, Museen, Sammlungen usw. arbeiten oder diese Institutionen besuchen, benutzen und unterstützen. Die Druckausgabe erscheint viermal jährlich. Jedes Heft ist schwerpunktmässig einem Thema gewidmet, wobei diese Themen so behandelt werden, dass die Mitglieder beider Berufsverbände und alle Sprachregionen der Schweiz angesprochen werden. Neben dem Editorial und den unter verschiedenen Rubrikenüberschriften versammelten Artikeln bringt arbido auch Nachrichten und Rezensionen. Im Interesse und auf Wunsch ihrer Mitglieder publizieren die beiden herausgebenden Vereinigungen die Zeitschrift parallel in elektronischer Form und stellen auch ein Archiv zurück bis zum Jahr 2000 online zur Verfügung. Die kostenlose Online-Ausgabe ist allerdings jeweils nur ein grosses PDF-File mit 
dem gesamten Inhalt des betreffenden Heftes. Die Redaktion unterteilt sich laut Impressum in eine „Redaktion BIS“ (vier Mitglieder) und eine „Redaktion VSA-AAS“ (zwei Mitglieder). Die Begutachtung der Beiträge, die nicht unter einer freien Lizenz erscheinen, erfolgt offenbar nur redaktionell. Im direkten Umfeld der Zeitschrift erscheinen jährlich auch acht bis zwölf Hefte eines elektronischen Newsletters.

Zuletzt führt der Streifzug in dieser Kategorie nach Südtirol. Zum Lesen: Fachzeitschrift für Südtiroler Bibliotheken heisst das dort vom Bibliotheksverband Südtirol in Zusammenarbeit mit dem Amt für Bibliotheken und Lesen (beide Bozen) herausgegebene, spartenübergreifende deutschsprachige Journal, das dreimal jährlich im Druck sowie parallel dazu kostenfrei online erscheint. Wie schon im Fall einiger oben besprochener Zeitschriften ist auch hier die Online-Ausgabe jeweils ein PDF-File, das das betreffende Heft zur Gänze enthält. Das Online-Archiv solcher Files reicht bis 2002 zurück. Das aktuelle Redaktionsteam umfasst sechs Personen. Die Hefte haben in der Regel Themenschwerpunkte, bringen daneben aber auch andere Beiträge sowie Tagungsberichte, Mitteilungen und Buchbesprechungen. Ein Peer Review im engeren Sinn dürfte nicht implementiert sein; freie Lizenzen kommen nicht zur Anwendung.

\section{Zeitschriften mit zeitverzögertem offenen Zugang}

Von „delayed open access“ wird gesprochen, wenn Artikel aus Subskriptionszeitschriften von den betreffenden Verlagen nach Ablauf einer Sperrfrist online frei zugänglich gemacht werden. ${ }^{17}$ Dies kann sowohl alle oder nur bestimmte Artikel eines Zeitschriftenheftes betreffen. „Offen“ ist dabei meist nur der Zugang für die Endbenutzer/innen, wogegen die Artikel nicht unter eine freie Lizenz gestellt werden. Aus Endbenutzersicht ist natürlich vor allem die Länge des Embargos das entscheidende Kriterium, weshalb auch die folgende Übersicht danach geordnet ist. (s.a. Tab. 2).

Den kürzesten Sperrzeitraum weist die vom Berufsverband Information Bibliothek (Reutlingen) herausgegebene Zeitschrift BuB: Forum Bibliothek und Information auf, die zehnmal jährlich erscheint. Drei Monate nach Publikation der Druckausgabe sind die ganzen Hefte - jeweils als ein grosses, aber mit navigationserleichternden Ankern versehenes PDFFile - online frei zugänglich. Mitunter werden einzelne Beiträge auch schon bei Erscheinen frei verfügbar gemacht - es liegt also die oben erwähnte Mischform „some articles open access and the other delayed access" vor. Das Online-Archiv reicht bis zum Jahr 2006 zurück. BuB ist 
spartenübergreifend angelegt, allerdings mit (gefühltem) Übergewicht des ÖB-Sektors. Die neben Berichten, Rezensionen, Mitteilungen und Meldungen enthaltenen Artikel sind in der Regel eher kurz gehalten und praxisorientiert.

Zwei Journale sind erst nach einem sechsmonatigen Embargo offen zugänglich. Bei der ersten handelt es sich um Dialog mit Bibliotheken, die zweimal jährlich erscheinende Fachzeitschrift der Deutschen Nationalbibliothek. Sie bringt zwar vor allem Beiträge über diese Institution, ihre Aktivitäten und ihr Dienstleistungsangebot, ist aber für die Bibliothekspraxis im gesamten deutschsprachigen Raum von Interesse und Relevanz. Die Online-Ausgabe, die offenbar auch für Abonnenten nicht früher verfügbar ist, besteht jeweils aus einer einzigen grossen PDF-Datei. Das Volltext-Archiv geht bis Ausgabe 2/2006 zurück, das Archiv der Inhaltsverzeichnisse bis 2002 (als die Zeitschrift noch dreimal jährlich erschien).

Auch die Fachzeitschrift des Verbandes der Bibliotheken des Landes Nordrhein-Westfalen, ProLibris, ist erst nach einer Frist von sechs Monaten frei elektronisch verfügbar. Das von diesem Verband sowie den Dezernaten für Öffentliche Bibliotheken der Bezirksregierungen herausgegebene Journal erscheint viermal im Jahr und wird seit 2015 an die Verbandsmitglieder kostenlos abgegeben. Die Online-Ausgabe - ebenfalls immer nur ein grosses PDF-File - steht aber auch diesen sowie zahlenden Abonnenten erst zeitversetzt zur Verfügung. Bis 1996 zurück können derartige Dateien abgerufen werden. In inhaltlicher Hinsicht ist ProLibris spartenübergreifend, wobei jedoch der ÖB-Bereich zu dominieren scheint. Die Hefte weisen meist einen thematischen Schwerpunkt auf. Neben der Redakteurin wird im Impressum auch ein aus fünf Personen bestehendes Herausgebergremium angeführt, dem vermutlich die Begutachtung der Artikel obliegt.

Leider erst nach einer zwölfmonatigen Sperrfrist sind die im Verlag De Gruyter erscheinenden Fachzeitschriften aus dem Gebiet Bibliothek und Information online frei zugänglich. Es handelt sich um bekannte und daher hier nicht detaillierter zu behandelnde Titel:

- ABI-Technik

- Bibliothek: Forschung und Praxis

- Bibliotheksdienst

- Information - Wissenschaft und Praxis.

Für Abonnenten steht die jeweilige Online-Ausgabe ohne Zeitverzug zur Verfügung. Das Online-Interface des Verlags ist relativ komfortabel und verleiht den vier Zeitschriften einen einheitlich anmutenden Webauftritt. Die Archive der früheren Jahrgänge bei De Gruyter reichen unterschiedlich 
weit zurück (in der obigen Reihenfolge bis 1996, 1977, 1967, 2012). Im Fall der Zeitschrift Bibliotheksdienst hat die vor einigen Jahren erfolgte Übernahme durch den Verlag bedauerlicherweise zu einer Vervierfachung der Wartezeit für Benutzer/innen geführt, da zuvor ein Grossteil der Artikel bereits drei Monate nach Erscheinen auf dem Server der Zentral- und Landesbibliothek Berlin frei zugänglich war.

Als weiteres bekanntes Verlagsprodukt mit einer einjährigen Sperrfrist ist die Zeitschrift für Bibliothekswesen und Bibliographie (ZfBB) zu nennen, die im Verlag Klostermann erscheint. Wenn die in Kooperation mit der Thüringer Universitäts- und Landesbibliothek Jena produzierte Online-Version über den Anschluss einer Institution aufgerufen wird, die $Z f B B$ abonniert hat, besteht gleich nach Erscheinen Zugriff auf die Volltexte der Zeitschrift, sonst erst nach zwölf Monaten. Das Online-Interface ist m.E. weniger komfortabel als im Fall der obigen vier Journale; das Online-Archiv reicht bis 2006 zurück.

\begin{tabular}{|c|c|c|c|}
\hline Zeitschrift & Web-Adresse & $\begin{array}{l}\text { Ausgaben/ } \\
\text { Jahr }\end{array}$ & Sperrfrist \\
\hline $\begin{array}{l}\text { BuB: Forum Bibliothek } \\
\text { und Information }\end{array}$ & http://www.b-u-b.de/ & 10 & 3 Monate \\
\hline Dialog mit Bibliotheken & http://www.dnb.de/dialog & 2 & 6 Monate \\
\hline ProLibris & $\begin{array}{l}\text { http://www.bibliotheken-nrw.de/zeitschrift- } \\
\text { prolibris/ }\end{array}$ & 4 & 6 Monate \\
\hline ABI-Technik & http://www.degruyter.com/view/j/abitech & 4 & 12 Monate \\
\hline $\begin{array}{l}\text { Bibliothek: Forschung } \\
\text { und Praxis }\end{array}$ & http://www.degruyter.com/view/j/bfup & 3 & 12 Monate \\
\hline Bibliotheksdienst & http://www.degruyter.com/view/j/bd & 12 & 12 Monate \\
\hline $\begin{array}{l}\text { Information - } \\
\text { Wissenschaft und Praxis }\end{array}$ & http://www.degruyter.com/view/j/iwp & 6 & 12 Monate \\
\hline $\begin{array}{l}\text { Zeitschrift für } \\
\text { Bibliothekswesen und } \\
\text { Bibliographie }\end{array}$ & $\begin{array}{l}\text { http://zs.thulb.uni-jena.de/receive/jportal_jp- } \\
\text { journal } 00000014\end{array}$ & 6 & 12 Monate \\
\hline APBB aktuell & http://www.apbb.de/weblog/?page_id=1090 & $?$ & 12 Monate \\
\hline Auskunft & $\begin{array}{l}\text { http://resolver.sub.uni-hamburg.de/goobi/ } \\
\text { PPN326504192 }\end{array}$ & 2 & 5 Jahre \\
\hline BIT online & http://www.b-i-t-online.de/ & 6 & unbekannt \\
\hline
\end{tabular}

Tab. 2: Zeitschriften mit zeitverzögertem bzw. partiellem offenen Zugang 
Handelte es sich bei den bisher in diesem Abschnitt besprochenen Journalen durchwegs um hybride Publikationen, so ist die von der Arbeitsgemeinschaft der Parlaments- und Behördenbibliotheken herausgegebene Zeitschrift APBB aktuell: Behörde - Bibliothek - Information eine reine OnlinePublikation mit zeitverzögertem offenen Zugang. Die Zeitschrift ging 2013 aus zwei Vorgängerpublikationen hervor. Sie kann in den ersten zwölf Monaten nach Erscheinen nur im Mitgliederbereich aufgerufen werden und ist erst nach Ablauf dieser Frist - als Gesamt-PDF-Datei - öffentlich zugänglich. Mit Stand Mai 2015 war allerdings erst ein Heft abrufbar (Nr. 64, Mai 2013).

Bereits im 35. Jahrgang erscheint das im Auftrag des Landesverbandes Hamburg im Deutschen Bibliotheksverband und des Landesarchivs Schleswig-Holstein herausgegebene Journal Auskunft: Zeitschrift für Bibliothek, Archiv und Information in Norddeutschland mit zwei Heften pro Jahr im Verlag Bautz (Nordhausen). Das auf einem Server der SUB Hamburg frei zugängliche Online-Archiv reicht bis zum ersten Jahrgang zurück, endet aber derzeit mit dem Jahr 2011. Laut EZB sind nämlich die aktuellen fünfJahrgänge [sic!] nicht im Volltext online verfügbar. ${ }^{18}$ Tatsächlich weist eine Recherche im Katalog der SUB Hamburg als letzten erschienen Band den Jahrgang 2014 nach, sodass die Zeitschrift wohl durchaus zu den „lebenden“ Periodika zu rechnen ist.

\section{Zeitschriften mit teilweisem offenen Zugang}

Die Überschrift zu diesem Abschnitt steht nur deshalb im Plural, da es sich dabei gleichsam um eine Kategorienbenennung handelt. In der Tat konnte nämlich nur ein Journal eruiert werden, das zurzeit in diese Kategorie fällt. Es handelt sich um die bekannte Zeitschrift BIT online: Bibliothek, Information, Technologie, ein hybrides Journal, das im Verlag Dinges \& Frick erscheint und dessen vollständige Online-Version nur für Abonnenten zugänglich ist. Ein variierender Anteil der Beiträge ist aber auch für das gesamte Publikum frei verfügbar, ein anderer Teil nicht. Die nicht frei verfügbaren Teile der Zeitschrift bleiben wohl auch für längere Zeit oder gar für immer gesperrt Genaueres ist nicht ohne weiteres eruierbar (vermutlich liegt aber doch der Mischtyp „some articles open access and the other delayed access" vor). Ein Online-Archiv der früheren Ausgaben wird jedenfalls zurück bis zum zweiten Jahrgang von BIT online (1999) vorgehalten. Eine Begutachtung der Fachbeiträge findet statt, ist meiner Einschätzung nach aber eher ein editorial review als ein klassisches peer-review. 


\section{Schlussbemerkungen}

Schliesslich gibt es auch eine (kleine) Gruppe von Zeitschriften aus dem Themenbereich Bibliothek und Information, die gar nicht offen zugänglich sind. Die bekannteste davon ist wohl Password: Nachrichten \& Wissen für Informationsprofis im deutschsprachigen Raum, ${ }^{19}$ die sich mit elf Print- und Onlineausgaben pro Jahr an Anbieter und Nutzer elektronischer Informationsdienste im deutschsprachigen Raum wendet und verschiedene Abonnement-Varianten anbietet. Letzteres trifft auch auf die zehnmal pro Jahr online und im Druck erscheinende Zeitschrift Library Essentials ${ }^{20} z u$, die erst seit 2012 erscheint und Fakten und Berichte für Informationsspezialisten bringt. Die von der EZB ebenfalls unter der Rubrik „Informations-, Buchund Bibliothekswesen, Schrift- und Handschriftenkunde" gelistete und nur für Abonnenten zugängliche Zeitschrift Wissensmanagement: Das Magazin für Führungskräfte ${ }^{21}$ dürfte hingegen eher zum Fachgebiet Betriebswirtschaft zu zählen sein.

Der Anteil der deutschsprachigen Fachzeitschriften für Bibliothek und Information mit vollständigem, zeitverzögertem oder partiellem offenen Zugang ist, allgemein betrachtet, erfreulich hoch. Dass gerade die bekanntesten und (vielleicht auch) bedeutendsten davon erst nach einer längeren Wartezeit frei verfügbar sind, ist natürlich weniger erfreulich. Die reinen Online-Journale kommen dem OA-Gedanken am nächsten oder erfüllen sogar alle OA-Kriterien. Die hybriden Zeitschriften mit vollständigem offenen Zugang sowie die nur zeitverzögert bzw. partiell offen zugänglichen Journale bieten zwar den freien Zugriff, nehmen aber in der Regel davon Abstand, ihrem Publikum weitere Nutzungsrechte (wie die eingangs erwähnten: freie Nach- und Weiternutzung, Vervielfältigung, Verbreitung oder auch Veränderung der Dokumente) unter einer freien Lizenz einzuräumen. Nur in Einzelfällen wird die auf den Webseiten dieser Journale auf die oben erwähnte Erlaubnis zur Selbstarchivierung eingegangen. So heisst es etwa in den Hinweisen für Autoren der Zeitschrift Bibliotheksdienst: „Entsprechend Vereinbarung mit dem Verlag können Autoren ihre Artikel (als Verlags-PDF) 6 Monate nach Erscheinen über die eigene Website oder ihr hochschuleigenes Institutional Repository der Öffentlichkeit zur Verfügung stellen." 22

Im vorliegenden Beitrag wurde lediglich der mit OA primär verbundene Aspekt des entgeltfreien Zugangs zu wissenschaftlicher (bzw. professioneller) Information untersucht. Die Betrachtung der Finanzierung von OA-Publikationen - auch „Geschäftsmodell“ (business model) genannt ${ }^{23}$ - blieb ganz bewusst „aussen vor“. Im Hinblick auf die stets mögliche Dop- 
pelrolle von Leser/innen der Zeitschriften des Bibliotheks- und Informationswesens als Autor/innen dieser Journale sei aber zumindest festgehalten, dass sich in unserem Bereich die Umwälzung der Kosten für OA auf die letzteren („,author-pays-model“, „article processing charges“) bisher nicht etabliert hat.

Dr. Otto Oberhauser MPhil MSc MLIS

E-Mail: oberh@web.de

Website: http://oco.vfi-online.org GND-IDNr.: 110343921

1 http://www.budapestopenaccessinitiative.org/boai-10-translations/ german-translation [22.07.2015]

2 https://doaj.org/faq [22.07.2015]

3 https://doaj.org/application/new [22.07.2015] (dort Punkt 21)

4 http://open-access.net/informationen-zu-open-access/open-accesszeitschriften/ [10.05.2015; Hervorhebungen d. Verf.]

5 http://open-access.net/informationen-fuer-verschiedene-zielgruppen/ autorinnen/ [22.07.2015]. Zu den im Bereich von Wissenschaft und Forschung bedeutsamen Creative Commons-Lizenzen (CC) vgl. die Informationen auf der Seite https://creativecommons.org/licenses/ [10.05.2015].

6 https://en.wikipedia.org/wiki/Open_access_journal [22.07.2015]

7 Aus Sicht der OA-Community sind das zwar Spielarten von Open Access, aber keine „Open Access Journals“. Als solche gelten nur jene, die $100 \%$ ihrer Inhalte ohne Einschränkungen anbieten und somit den

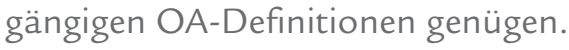

8 http://ezb.uni-regensburg.de/fl.phtml?bibid=UBR\&colors=7\&lang= de\&notation=AN [10.05.2015; gleichfalls alle nachfolgend zitierten Links]

9 Oberhauser, O. (2014). Open Access-Zeitschriften für Bibliothek und Information: Ein Überblick über die jüngste Entwicklung. Mitteilungen der Vereinigung Österreichischer Bibliothekarinnen und Bibliothekare. 67(2). 262-271. Online: http://hdl.handle.net/10760/23851

10 Auch im vorliegenden Beitrag wird gelegentlich Text aus den Webseiten der betreffenden Zeitschriften übernommen oder paraphrasiert, ohne dass dies jeweils explizit ausgewiesen wird.

11 http://www.ub.uni-dortmund.de/listen/inetbib/msg54615.html

$12 \mathrm{Vgl}$.: http://www.vdb-online.org/publikationen/vdb-mitteilungen/ 
13 Da der vorliegende Beitrag nur auf den im Web verfügbaren Informationen basiert, kann hier lediglich eine Vermutung geäussert werden. Dies gilt analog auch bei einigen nachfolgend besprochenen Zeitschriften, auf deren Webseiten keine genaueren Details zu finden waren.

14 Der Terminus „hybrid“ wird hier für die Kombination aus Print und Online verwendet (und nicht im Sinne von einzelnen freigekauften Open Access-Artikeln in Subskriptionszeitschriften).

15 http://www.qucosa.de

16 Bspw. ist das aktuelle Heft sowohl Jg. 10, H.1, als auch die 28. Ausgabe.

17 Vgl. z.B.: Laakso, M.; Björk, B.-C. (2013). Delayed Open Access - an overlooked high-impact category of openly available scientific literature. Journal of the American Society for Information Science and Technology. 64(7). 1323-1329.

18 http://ezb.uni-regensburg.de/detail.phtml?bibid=UBR\&colors=7\&lang =de\&jour id=215361

19 http://www.password-online.de

20 http://www.libess.de

21 http://www.wissensmanagement.net/

22 http://www.degruyter.com/view/supplement/s21949646 Autorenhinweise.pdf

$23 \mathrm{Vgl}$ z.B.: http://open-access.net/informationen-zu-open-access/geschaeftsmodelle/

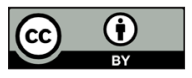

Dieses Werk ist lizenziert unter einer Creative-Commons-Lizenz Namensnennung 4.0 International 